Check for updates

Cite this: RSC Adv., 2018, 8, 14760

\title{
Persistent radical anion polymers based on naphthalenediimide and a vinylene spacer $\dagger$
}

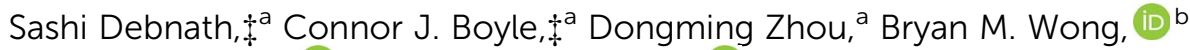 \\ Kevin R. Kittilstved (D) and D. Venkataraman (D)*a
}

Persistent $\mathrm{n}$-doped conjugated polymers were achieved by doping the electron accepting PDNDIV and PFNDIV polymers with ionic (TBACN) or neutral (TDAE) dopants. The great electron affinities, as indicated by the low LUMO levels of PDNDIV $(-4.09 \mathrm{eV})$ and PFNDIV $(-4.27 \mathrm{eV})$, facilitated the chemical reduction from either TBACN or TDAE. The low-lying LUMOs of the neutral polymers PDNDIV and PFNDIV were achieved by incorporation of vinylene spacers between the electron poor NDI units to increase the conjugation length without the use of an electron donor, and this was lowered further by an electron-withdrawing fluorinated $\mathrm{N}$-substituent on the NDI moiety. The polymer radical anions were found to persist for several days under ambient conditions by EPR spectroscopy. A distinguishing and noteworthy feature of these polymers is that they can be consecutively reduced by up to four electrons in acetonitrile. Conductivity measurements demonstrate the prospective impact of PDNDIV and PFNDIV for organic electronics.

Received 19th March 2018

Accepted 12th April 2018

DOI: $10.1039 / \mathrm{c} 8 \mathrm{ra02417k}$

rsc.li/rsc-advances

naphthalenediimide-based polymers that can be n-doped under ambient conditions. We also demonstrate the important role of side chains in tuning and enhancing the air-stability of the doped polymers.

We had previously reported a straightforward route to electron-transporting polymers from readily available electron accepting monomers and vinylene spacers. ${ }^{7}$ Using this route, and monomers containing naphthalene diimide (NDI) units, we designed two new naphthalene diimide-vinylene (NDIV) copolymers. We chose to incorporate vinylene spacers instead of electron-rich donor units since vinylene groups impose planarity of the conjugated polymer while maintaining a high electron affinity. We chose to utilize two NDI monomers, one with alkyl hydrocarbon side chains on both imide nitrogens $\left[N, N^{\prime}\right.$-bis $(n-$ dodecyl)-2,6-dibromo-1,4,5,8-naphthenedicarboximide (DNDI), Scheme 1] and the other with an alkyl fluorocarbon on one imide nitrogen and an alkyl hydrocarbon chain on the other imide nitrogen $\quad\left[N-(2,2,3,3,4,4,4\right.$-heptafluorobutyl $)-N^{\prime}$-octadecyl-2,6dibromo-1,4,5,8-naphthenedicarboximide (FNDI), Scheme 1]. We chose naphthalene diimide because it has been shown to form stable radical anions. ${ }^{8-10}$ Based on the work by Katz and coworkers, ${ }^{\mathbf{1 1}}$ we hypothesized that the fluorocarbon sidechain will lower the lowest unoccupied molecular orbitals (LUMO), increase the electron affinity, and thus chemical stability of the n-doped polymer. To shed additional insight into the electronic properties of these systems, we carried out first-principles density functional theory (DFT) calculations on a series of oligomers ranging from 1 to 6 monomer units for both the FNDI and DNDI systems. Both the electron affinity and the spin density (see Fig. S1 in the ESI for details $\dagger$ ) were computed at the $\omega \mathrm{B} 97 \mathrm{XD} / 6$ -

${ }^{a}$ Department of Chemistry, University of Massachusetts Amherst, 710 North Pleasant Street, Amherst, Massachusetts 01003,USA.E-mail: dv@chem.umass.edu

${ }^{b}$ Department of Chemical \& Environmental Engineering and Materials Science \& Engineering Program, University of California, Riverside, 900 University Avenue, Riverside, CA 925521, USA

$\dagger$ Electronic supplementary information (ESI) available. See DOI: 10.1039/c8ra02417k

\$ These authors equally contributed to this work. 


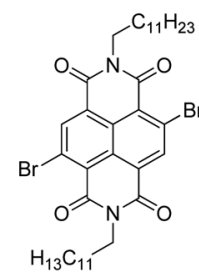

DNDI<smiles>CC(C)CN1C(=O)c2cc(Br)c3c4c(c(Br)cc(c24)C1=O)C(=O)N(CC(C)(F)F)C3=O</smiles>

FNDI

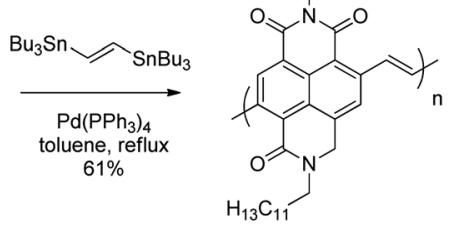

PDNDIV

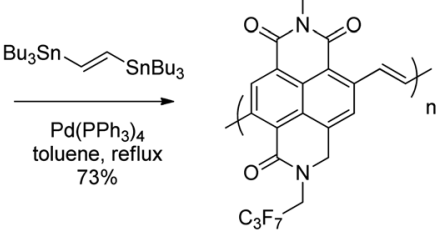

PFNDIV
Scheme 1 Synthesis of PDNDIV and PFNDIV by Stille coupling reaction.

$31 \mathrm{~g}(\mathrm{~d}, \mathrm{p})$ level of theory, ${ }^{12}$ which has been previously benchmarked for a variety of charged, conjugated organic systems. ${ }^{13,14}$ Based on our DFT calculations, we found that oligomers with FNDI had an electron affinity that was $0.2 \mathrm{eV}$ higher than oligomers with DNDI.

The polymers, poly $\left[N, N^{\prime}\right.$-bis( $n$-dodecyl)-1,4,5,8-naphthalenedicarboximide-2,6-diylvinylene] (PDNDIV) and poly[ $N$-(2,2,3,3,4,4,4heptafluorobutyl)- $N^{\prime}$-octadecyl-1,4,5,8-naphthalenedicarboximide2,6-diylvinylene] (PFNDIV) were synthesized using previously reported methods. ${ }^{7,15,16}$ The polymers were doped by chemical reduction using either tetrabutylammonium cyanide (TBACN) $)^{17-19}$ or tetrakis(dimethylamino)ethylene (TDAE). ${ }^{5,20}$ Consistent with our hypothesis, we found that the incorporation of a fluorinated side chain in PFNDIV increased the electron affinity, as indicated by its decreased LUMO energy level $(-4.27 \mathrm{eV})$, estimated from electrochemical measurements. Each polymer showed well-defined spectroscopic changes after gradual addition of either dopant due to the formation of radical anions, which were confirmed by EPR. Both of the doped polymers exhibit signatures of the radical anion for several days in THF under ambient conditions. We also found a distinguishing and noteworthy feature of these polymers: they can be consecutively reduced by up to four electrons in acetonitrile.

\section{Results and discussion}

\section{Synthesis and characterization}

The synthetic methods for DNDI, FNDI, PDNDIV, and PFNDIV are shown in Scheme 1, and the experimental procedures are described in the ESI. $\uparrow$ The molecular weights of the polymers were measured by high temperature gel permeation chromatography (GPC) using 1,2,4-trichlorobenzene as the mobile phase and summarized in Table 1.

The thermal stability of PDNDIV and PFNDIV was determined by using thermogravimetric analysis (TGA) with a heating rate of $10^{\circ} \mathrm{C} \mathrm{min}^{-1}$ under nitrogen atmosphere (Fig. S2a†).
Table 1 Physical properties of the polymers

\begin{tabular}{ll}
\hline Polymer & $M_{\mathrm{n}}\left(\mathrm{g} \mathrm{\textrm {mol } ^ { - 1 } )}\right.$ \\
\hline PDNDIV & 10622 \\
PFNDIV & 8304
\end{tabular}

TGA curves showed that the decomposition temperatures $\left(T_{\mathrm{d}}\right.$, at which 5\% weight loss occurred) for PDNDIV and PFNDIV were found to be 239 and $279{ }^{\circ} \mathrm{C}$, suggesting sufficient thermal stability of the polymers (Table 1). Differential scanning calorimetry (DSC, see Fig. S2b and S2c $\dagger$ ) and powder X-ray diffraction patterns (Fig. S3 $\dagger$ ) of the polymers did not show any distinguishing peaks, which indicates that the solid-state structure of the polymers are amorphous.

\section{Optoelectronic properties}

The UV-Vis absorption spectra of each polymer in dilute chloroform solution $\left(10^{-5} \mathrm{M}\right.$ on the basis of repeating units) and of thin films on glass substrates coated with indium tin oxide (ITO) is shown in Fig. 1a. Both polymers showed two distinctive absorption bands, one in the blue region from $300-400 \mathrm{~nm}$ and the other in the red region $450-650 \mathrm{~nm}$. We attribute these absorption to $\pi-\pi^{*}$ transitions of the NDI chromophore and electronic transitions between new delocalized bands in the polymer backbone, ${ }^{21}$ respectively (Table 2). A distinct broadening and red shift of the low energy transitions by $10-20 \mathrm{~nm}$ is observed in the polymer films, which indicates chromophore aggregation caused by interactions between the chains in the solid-state. ${ }^{22}$ The optical band gaps $\left(E_{\mathrm{g}}\right)$ obtained from the Tauc plot (see Fig. 1a inset) are $2.11 \mathrm{eV}$ for PDNDIV and $2.04 \mathrm{eV}$ for PFNDIV. The reduced $E_{\mathrm{g}}$ value of PFNDIV compared to that of PDNDIV was attributed to the presence of the electronwithdrawing fluorinated side chain, which can lower the LUMO energy level through inductive effects. ${ }^{11}$

$$
\begin{gathered}
E_{\mathrm{LUMO}}=-4.8 \mathrm{eV}-\left(E_{\text {reduction }}-0.32 \mathrm{eV}\right) \\
E_{\mathrm{HOMO}}=E_{\mathrm{LUMO}}-E_{\mathrm{g}}
\end{gathered}
$$
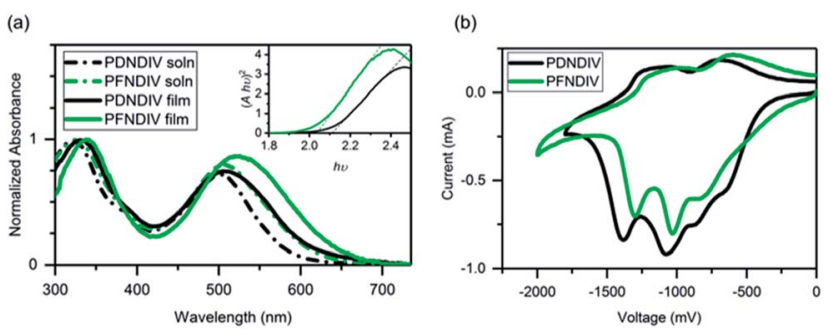

Fig. 1 (a) UV-Vis spectra of the polymers in $10^{-5} \mathrm{M}$ chloroform solution and as thin films on ITO coated glass. The Tauc plot of the film spectra, assuming $r=1 / 2$ for a direct allowed transition, including the dashed trace used to determine the bandgap is inset. (b) Cyclic voltammograms of the polymer film dropcast onto a Pt disk $(1.6 \mathrm{~mm}$ diameter) electrode as the working electrode, using Pt wire as the counter electrode, $\mathrm{Ag} / \mathrm{AgCl}$ as the reference electrode, and $0.1 \mathrm{M}$ tetrabutylammonium perchlorate as the supporting electrolyte at a scan rate of $50 \mathrm{mV} \mathrm{s}^{-1}$, reduction only scans. 
Table 2 Optical and electrochemical properties of the polymers

\begin{tabular}{llllll}
\hline Polymer & $\begin{array}{l}\lambda_{\max } \\
\text { solution }(\mathrm{nm})\end{array}$ & $\lambda_{\max }$ film $(\mathrm{nm})$ & $E_{\text {reduction }}(\mathrm{eV})$ & ${ }^{a} E_{\text {HOMO }}(\mathrm{eV})$ & ${ }^{b} E_{\text {LUMO }}(\mathrm{eV})$ \\
\hline PDNDIV & 322,500 & 330,509 & -0.392 & -6.20 & -4.09 \\
PFNDIV & 325,506 & 338,524 & -0.208 & -6.31 & -4.27
\end{tabular}

${ }^{a}$ Eqn (2). ${ }^{b}$ Eqn (1). ${ }^{c}$ Obtained from the Tauc plot.

Cyclic voltammograms (CV) of the polymer films are shown in Fig. 1b. There were multiple reduction onsets in the CV, indicating that polymers can be consecutively reduced by up to 4 electrons in acetonitrile. LUMO energies of both the polymers were calculated from their first onset reduction potentials (eqn (1)) as $-4.09 \mathrm{eV}$ for PDNDIV and $-4.27 \mathrm{eV}$ for PFNDIV. The HOMO energy levels were estimated by subtracting the $E_{\mathrm{g}}$ from the above CV-determined LUMO energy levels and were found to be $-6.20 \mathrm{eV}$ for PDNDIV and $-6.31 \mathrm{eV}$ for PFNDIV (eqn (2)). CV data showed that both polymers are positioned at low-lying LUMO levels and the electron withdrawing $N$-substituents impart a significant impact on PFNDIV's superior $\pi$-acidity, which is evident from additional diminution in reduction potential.

\section{Characterization and stability study of radical anion polymers}

The polymers were doped using TDAE, a neutral electron donor, or TBACN, an anionic electron donor, under ambient conditions. TDAE ${ }^{17-19}$ and $\mathrm{TBACN}^{5,20}$ have been used as an electron donors for rylene diimides, and their proposed mechanism of doping is a single electron transfer. In the UV-Vis-NIR spectra, we found that the continuous addition of $0-1.4$ equivalents (with respect to the polymer's repeating units) of TBACN with PFNDIV, progressively bleached the neutral polymer absorption band $(450-650 \mathrm{~nm})$ and simultaneously produced a new absorption band at $750 \mathrm{~nm}$, with an isosbestic point near $600 \mathrm{~nm}$ (Fig. 2a). A similar behavior was observed for PDNDIV, but with larger numbers of equivalents $(0-10)$ of TBACN (Fig. $2 \mathrm{~b}$ ) required to produce similar spectral changes.

The formation of a weaker absorption band at $\sim 750 \mathrm{~nm}$ suggests the formation of the radical anion polymers [PDNDIV $]^{\cdot-}$

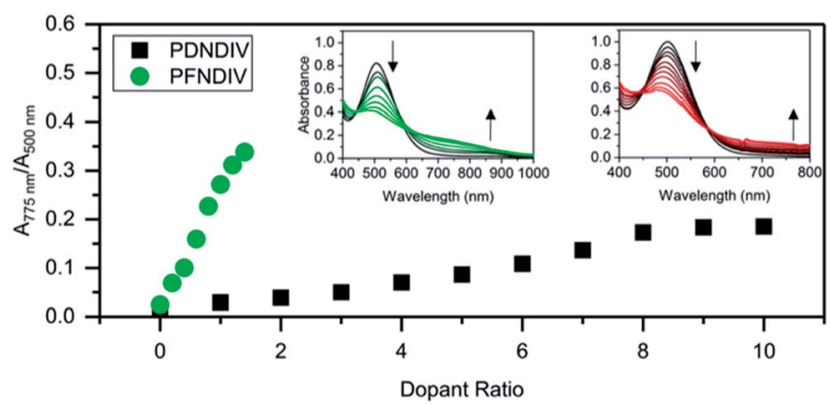

Fig. 2 Ratio of doped to undoped repeating units, estimated by UVVis-NIR spectroscopic changes, for PDNDIV and PFNDIV in THF with increasing molar equivalents of TBACN; insets are the UV-Vis-NIR spectra for PFNDIV (left) and PDNDIV (right). and [PFNDIV $]^{\cdot-}$ (Fig. 3), which was also confirmed by EPR spectroscopy (vide infra). The addition of TDAE also resulted in an instantaneous color change of the polymer solution (inset Fig. S5 $\dagger$ ), which is consistent with an n-doped polymer. TDAEdoped radical anion polymers were also characterized by UVVis-NIR absorption (Fig. S5†) and EPR spectroscopy (Fig. S6b†).

EPR spectroscopy was used to probe the formation of a radical anion and monitor the relative stability of the radical under ambient conditions over time. The EPR spectra of the polymers after addition of TBACN are shown in Fig. 4. Both PDNDIV and PFNDIV display a single, narrow resonance at $g=$ 2.0035 upon mixing with TBACN, which indicates the formation of [PDNDIV $]^{\cdot-}$ and [PFNDIV $]^{\cdot-}$ radical anions. The absence of any hyperfine splitting indicates that the spin density of the radical anion is localized on the carbon of the aromatic ring. DFT calculations performed with the $\omega \mathrm{B} 97 \mathrm{XD} / 6-31 \mathrm{~g}(\mathrm{~d}, \mathrm{p})$ basis verify the nature of the SOMO as shown in the difference plots of the molecular orbital spin density (Fig. S1†). Both polymer radical anions are persistent as THF solutions stored in air, but the radical signal of [PFNDIV] ${ }^{--}$is more stable with an apparent half-life on the order of one week (Fig. 4b), comparable to the stability of the recently reported n-type polymer, ClBDPPV. ${ }^{6}$ The decay of the radical EPR signal is tentatively assigned to quenching by triplet oxygen. The difference in radical stabilities could possibly originate from variation in the reduction potentials of the LUMOs of [PDNDIV $]^{--}$and [PFNDIV $]^{\cdot-}$, with the more negative LUMO on PFNDIV. Interestingly, the EPR
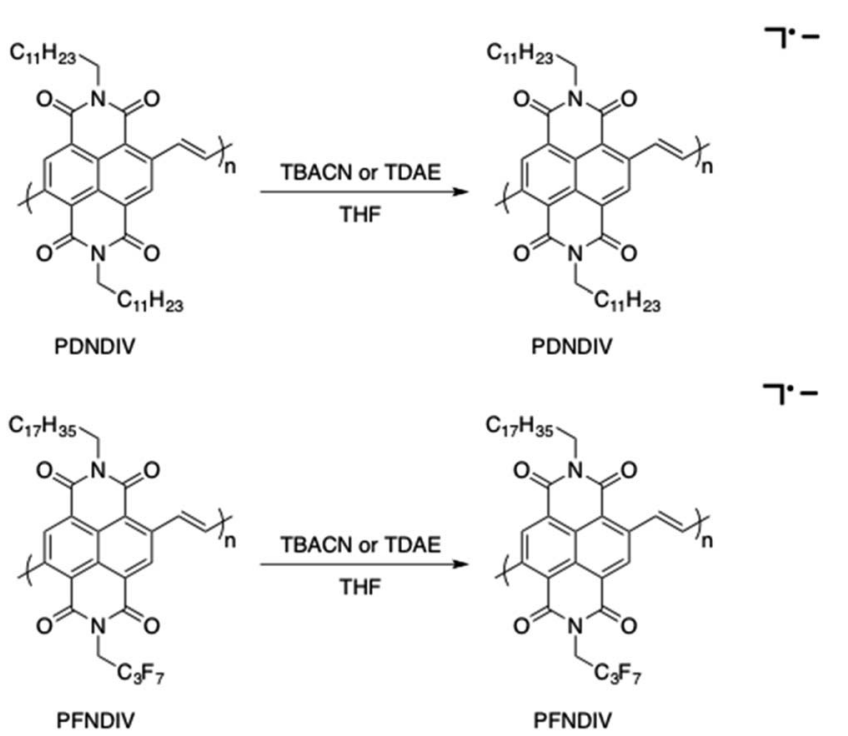

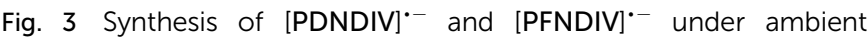
condition in THF using TBACN or TDAE. 

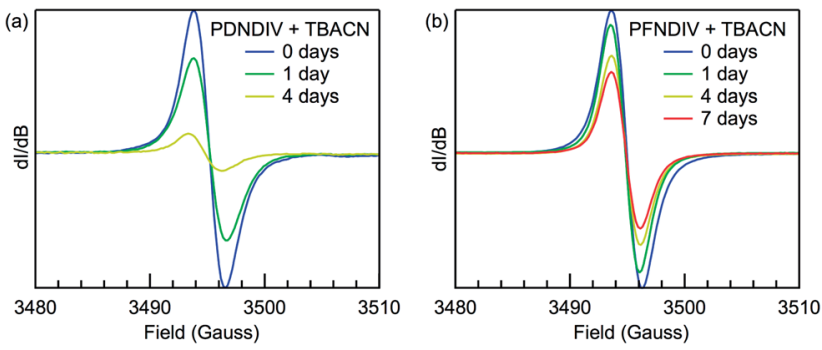

Fig. 4 Room-temperature X-band EPR spectra of (a) 2 mM (repeating units) PDNDIV with $10 \mathrm{mM}$ TBACN and (b) $2 \mathrm{mM}$ PFNDIV with $2 \mathrm{mM}$ TBACN in THF yielding [PDNDIV] ${ }^{\cdot-}$ and [PFNDIV $]^{\cdot-}$, respectively. The 7 day point for [PDNDIV] ${ }^{--}$is omitted and unreliable due to polymer precipitation.

spectrum of the as-prepared PFNDIV displays the same radical signal and suggests PFNDIV can be partially doped under ambient conditions while PDNDIV displays no signal (Fig. S6a†).

We then studied the impact of the concentration of TBACN on the intensity of the EPR signal Fig. 5. For PDNDIV we found that 1 equivalent of TBACN with respect to polymer repeating units was insufficient to dope the polymer. At higher concentrations, we observed an EPR signal, which indicated the formation of the radical anion. The intensity of the signal increased steadily with increasing concentration of the dopant. This observation is consistent with the presence of an equilibrium, with a slight preference for the reactants. This is consistent with the expected LUMO energy levels of PDNDIV and the cyanide anion of TBACN. In the case of PFNDIV, we found a steady increase in the EPR signal up to 1 equivalent of the TBACN. Further increase of the cyanide concentration led to a decrease in the EPR signal. From CV studies, we know that the
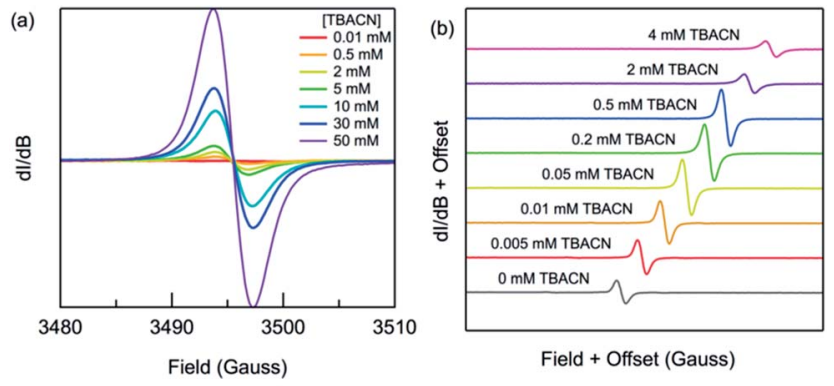

(c)

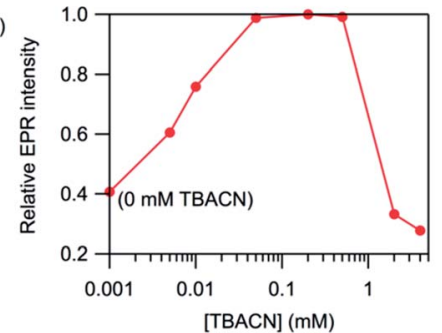

Fig. 5 Room-temperature X-band EPR spectra of (a) PDNDIV (2 mM) and (b) PFNDIV $(0.5 \mathrm{mM})$ after mixing with increasing concentration of TBACN in THF. (c) Normalized EPR intensity of PFNDIV $(0.5 \mathrm{mM})$ with increasing concentration of TBACN in THF. polymer can accept up to four electrons. Therefore, we tentatively attribute this decrease in [PFNDIV $]^{--}$EPR signal with increasing cyanide concentration to the formation of an EPRsilent singlet or triplet [PFNDIV $]^{2-}$ species.

\section{Conductivity studies}

To investigate the solid-state conductivity of these radical anion polymers under ambient conditions, we fabricated two-terminal devices using vacuum depositing Au electrodes (Fig. S11, $\uparrow$ inset) using neutral or doped PDNDIV or PFNDIV acting as the active layer. Either the neutral or doped polymers were drop-cast from THF on the fabricated channels, and current $(I)$-voltage $(V)$ measurements were carried out under ambient conditions (Fig. S9 and S10†). The conductivity of each doped polymer, 6.98 $\times 10^{-7} \mathrm{~S} \mathrm{~cm}^{-1}$ for [PDNDIV] ${ }^{--}$and $3.28 \times 10^{-7} \mathrm{~S} \mathrm{~cm}^{-1}$ for $[\text { PFNDIV }]^{--}$, was at least an order of magnitude greater than the conductivity of the neutral polymers, $3.49 \times 10^{-8} \mathrm{~S} \mathrm{~cm}^{-1}$ for PDNDIV and $2.61 \times 10^{-8} \mathrm{~S} \mathrm{~cm}^{-1}$ for PFNDIV. While these conductivities are lower than those of other reported n-doped conjugated polymers and are too low for practical applications, the drastic increase in conductivity upon doping and the air stability of the doped polymers suggests that these and similar NDI-vinylene polymers may find potential use as n-type organic electronic materials. The low conductivities may be due, in part, to the amorphous nature of these polymers in the solid state.

\section{Conclusions}

In summary, two new NDI-vinylene based electron acceptor polymers have been successfully synthesized. Due to the lowlying LUMOs $(-4.09$ and $-4.27 \mathrm{eV})$ both the polymers behave as strong $\pi$-acids. The electron-withdrawing effect of the fluorinated side chain reduces the LUMO level of PFNDIV significantly. Multistep reduction potentials indicate the presence of several electron accepting sites in the polymer backbone. Chemical reduction from electron donors to the polymer generates persistent radical anion polymers with ambient stability. Time-dependent EPR spectroscopy showed the stability of the radical anions exists beyond several days. Finally, conductivity measurements showed the positive impact of chemical doping on the polymer's electrical conductivity, making these materials promising for organic electronic applications.

\section{Conflicts of interest}

There are no conflicts to declare.

\section{Acknowledgements}

The authors acknowledge the Department of Chemistry University of Massachusetts Amherst for research facilities. S. D. acknowledges IUSSTF for a research fellowship. B. M. W. acknowledges the National Science Foundation for the use of supercomputing resources through the Extreme Science and 
Engineering Discovery Environment (XSEDE), Project No. TGCHE150040.

\section{Notes and references}

1 R. A. Schlitz, F. G. Brunetti, A. M. Glaudell, P. L. Miller, M. A. Brady, C. J. Takacs, C. J. Hawker and M. L. Chabinyc, Adv. Mater., 2014, 26, 2825-2830.

2 K. Shi, F. Zhang, C.-A. Di, T.-W. Yan, Y. Zou, X. Zhou, D. Zhu, J.-Y. Wang and J. Pei, J. Am. Chem. Soc., 2015, 137, 6979-6982.

3 B. D. Naab, S. Zhang, K. Vandewal, A. Salleo, S. Barlow, S. R. Marder and Z. Bao, Adv. Mater., 2014, 26, 4268-4272.

4 S. Hwang, W. J. Potscavage Jr., Y. S. Yang, I. S. Park, T. Matsushima and C. Adachi, Phys. Chem. Chem. Phys., 2016, 18, 29199-29207.

5 S. Wang, H. Sun, U. Ail, M. Vagin, P. O. A. Persson, J. W. Andreasen, W. Thiel, M. Berggren, X. Crispin, D. Fazzi and S. Fabiano, Adv. Mater., 2016, 28, 10764-10771. 6 X. Zhao, D. Madan, Y. Cheng, J. Zhou, H. Li, S. M. Thon, A. E. Bragg, M. E. DeCoster, P. E. Hopkins and H. E. Katz, Adv. Mater., 2017, 29, 1606928.

7 G. Nagarjuna, A. Kokil, J. Kumar and D. Venkataraman, J. Mater. Chem., 2012, 22, 16091-16094.

8 S. Kumar, M. R. Ajayakumar, G. Hundal and P. Mukhopadhyay, J. Am. Chem. Soc., 2014, 136, 1200412010.

9 Q. Song, F. Li, Z. Wang and X. Zhang, Chem. Sci., 2015, 6, 3342-3346.

10 S. K. Keshri, S. Kumar, K. Mandal and P. Mukhopadhyay, Chem.-Eur. J., 2017, 23, 11802-11809.
11 H. E. Katz, A. J. Lovinger, J. Johnson, C. Kloc, T. Siegrist, W. Li, Y.-Y. Lin and A. Dodabalapur, Nature, 2000, 404, 478-481.

12 J.-D. Chai and M. Head-Gordon, Phys. Chem. Chem. Phys., 2008, 10, 6615-6620.

13 M. R. Golder, B. M. Wong and R. Jasti, Chem. Sci., 2013, 4, 4285-4291.

14 P. Li, B. M. Wong, L. N. Zakharov and R. Jasti, Org. Lett., 2016, 18, 1574-1577.

15 X. Liang, L. Tan, Z. Liu, Y. Ma, G. Zhang, L. Wang, S. Li, L. Dong, J. Li and W. Chen, Chem. Commun., 2017, 53, 4934-4937.

16 H. Zhang, Y. Xie, X. Chen, T. Jia, W. Huang, S. Luo, Q. Hou, R. Zeng and Z. Sun, J. Electrochem. Soc., 2017, 164, A290A294.

17 Y. Kumar, S. Kumar, S. Kumar Keshri, J. Shukla, S. S. Singh, T. S. Thakur, M. Denti, A. Facchetti and P. Mukhopadhyay, Org. Lett., 2016, 18, 472-475.

18 M. R. Ajayakumar, D. Asthana and P. Mukhopadhyay, Org. Lett., 2012, 14, 4822-4825.

19 M. R. Ajayakumar, P. Mukhopadhyay, S. Yadav and S. Ghosh, Org. Lett., 2010, 12, 2646-2649.

20 M. Fujitsuka, S. S. Kim, C. Lu, S. Tojo and T. Majima, J. Phys. Chem. B, 2015, 119, 7275-7282.

21 P. M. Alvey and B. L. Iverson, Org. Lett., 2012, 14, 2706-2709. 22 L. Ye, X. Jiao, H. Zhang, S. Li, H. Yao, H. Ade and J. Hou, Macromolecules, 2015, 48, 7156-7163. 